\title{
Intrinsic nucleus-targeted ultra-small metal- organic framework for the type I sonodynamic treatment of orthotopic pancreatic carcinoma
}

Tao Zhang ${ }^{1,2}$, Yu Sun ${ }^{1,2}$, Jing Cao ${ }^{1,2}$, Jiali Luo ${ }^{1,2}$, Jing Wang ${ }^{1,2}$, Zhenqi Jiang ${ }^{3^{*}}$ and Pintong Huang ${ }^{1,2^{*}}$ (D)

\begin{abstract}
Background: Sonodynamic therapy (SDT) strategies exhibit a high tissue penetration depth and can achieve therapeutic efficacy by facilitating the intertumoral release of reactive oxygen species (ROS) with a short lifespan and limited diffusion capabilities. The majority of SDT systems developed to date are of the highly $\mathrm{O}_{2}$-dependent type II variety, limiting their therapeutic utility in pancreatic cancer and other hypoxic solid tumor types.

Results: Herein, a nucleus-targeted ultra-small Ti-tetrakis(4-carboxyphenyl)porphyrin (TCPP) metal-organic framework (MOF) platform was synthesized and shown to be an effective mediator of SDT. This MOF was capable of generating large quantities of ROS in an oxygen-independent manner in response to low-intensity ultrasound (US) irradiation $\left(0.5 \mathrm{~W} \mathrm{~cm}^{-2}\right)$, thereby facilitating both type I and type II SDT. This approach thus holds great promise for the treatment of highly hypoxic orthotopic pancreatic carcinoma solid tumors. This Ti-TCPP MOF was able to induce in vitro cellular apoptosis by directly destroying DNA and inducing S phase cell cycle arrest following US irradiation. The prolonged circulation, high intratumoral accumulation, and nucleus-targeting attributes of these MOF preparations significantly also served to significantly inhibit orthotopic pancreatic tumor growth and prolong the survival of tumor-bearing mice following Ti-TCPP + US treatment. Moreover, this Ti-TCPP MOF was almost completely cleared from mice within 7 days of treatment, and no apparent treatment-associated toxicity was observed.
\end{abstract}

Conclusion: The nucleus-targeted ultra-small Ti-TCPP MOF developed herein represents an effective approach to the enhanced SDT treatment of tumors in response to low-intensity US irradiation.

Keywords: Type I sonodynamic therapy, Intrinsic nucleus-targeted, Hypoxia, Ultra-small metal-organic framework, Orthotopic pancreatic carcinoma

\section{Background}

Sonodynamic therapy (SDT) is a relatively non-invasive approach to treating a range of cancer types $[1,2]$. SDT combines the advantage of a high tissue penetration

\footnotetext{
*Correspondence: 7520200073@bit.edu.cn; huangpintong@zju.edu.cn ${ }^{1}$ Department of Ultrasound in Medicine, The Second Affiliated Hospital of Zhejiang University School of Medicine, No.88 Jiefang Road Shangcheng District, Hangzhou 310009, People's Republic of China ${ }^{3}$ Institute of Engineering Medicine, Beijing Institute of Technology, No. 5, South Street, Zhongguancun, Haidian District, Beijing 100081, People's Republic of China
}

Full list of author information is available at the end of the article depth with the ability to induce the generation of reactive oxygen species (ROS) in order to kill tumor cells [3-8]. While promising, most SDT approaches rely on an $\mathrm{O}_{2}$-dependent type II SDT modality, limiting their utility in solid tumors [9-11]. In contrast, type I SDT is more hypoxia-tolerant as it relies upon the generation of cytotoxic radicals and superoxide anions, which can better kill tumors under hypoxic conditions. Pancreatic tumors are often considered to be the most hypoxic of all tumor types on average, with an average $\mathrm{O}_{2}$ pressure of less than $2.5 \mathrm{mmHg}$ in up to $0-16 \%$ of tumor area as compared to $30-50 \mathrm{mmHg}$ in normal tissues [12-14]. Improving the original author(s) and the source, provide a link to the Creative Commons licence, and indicate if changes were made. The images or other third party material in this article are included in the article's Creative Commons licence, unless indicated otherwise in a credit line to the material. If material is not included in the article's Creative Commons licence and your intended use is not permitted by statutory regulation or exceeds the permitted use, you will need to obtain permission directly from the copyright holder. To view a copy of this licence, visit http://creativecommons.org/licenses/by/4.0/. The Creative Commons Public Domain Dedication waiver (http://creativeco mmons.org/publicdomain/zero/1.0/) applies to the data made available in this article, unless otherwise stated in a credit line to the data. 


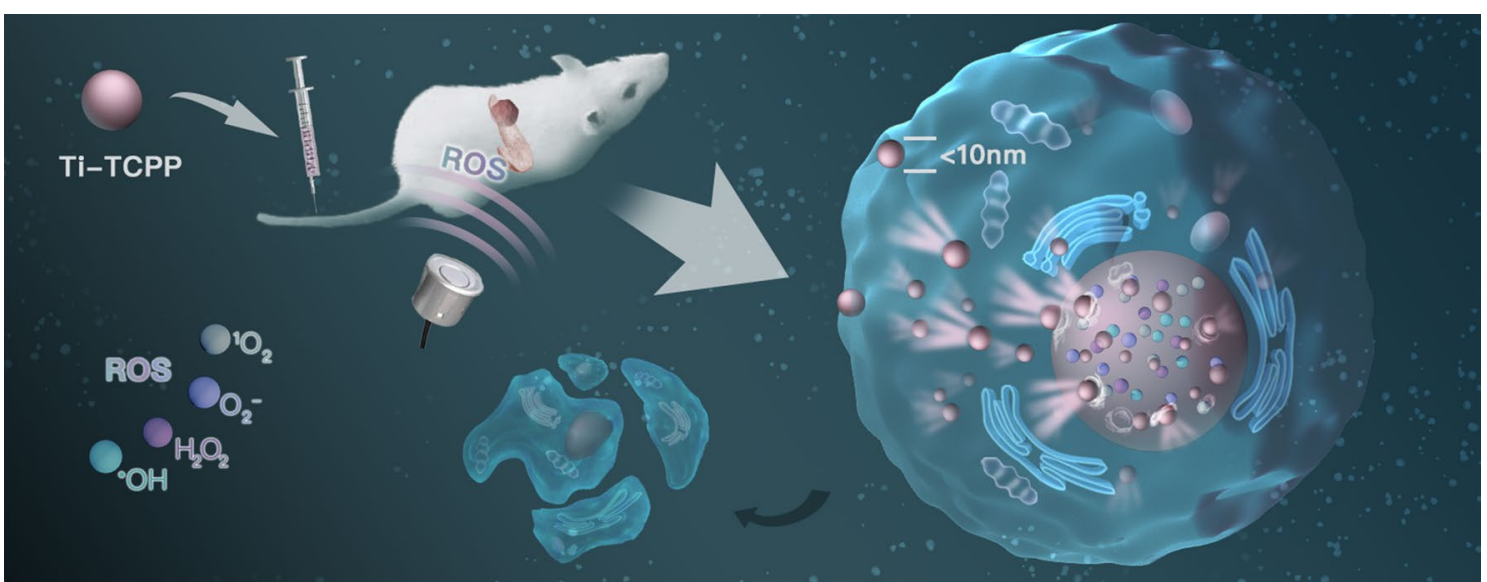

Scheme 1. A schematic illustration of ultra-small Ti-TCPP MOF application in nuclear-targeted SDT

utility of SDT in such hypoxic tumors is thus dependent upon the development of effective type I SDT strategies that can generate ROS under low $\mathrm{O}_{2}$ conditions $[15,16]$.

Another key determinant of SDT efficacy is the subcellular localization of sonosensitizing agents [17-19], as most generated ROS exhibit a very brief lifespan ( $<40 \mathrm{~ns})$ and a limited diffusion length $(\sim 20 \mathrm{~nm})[20,21]$. Recent evidence suggests that nanoagents located closer to DNA are better able to induce oxidative damage and to thereby achieve superior therapeutic efficacy [22-24]. This has led to efforts to target nanoparticles to cellular nuclei through approaches such as targeted design strategies and the utilization of particles with a positive surface charge [25]. Such targeting approaches typically rely on the modification of the type or density of surface ligands including peptides or adenoviral vectors so as to better target these particles to particular receptors that are expressed in cells of interest [21, 26, 27]. However, targeted aggregation within the nucleus can be limited by several factors, with the size of the nanoparticle being the most commonly studied of these limitations. It has been reported that nanoparticles smaller than $50 \mathrm{~nm}$ in diameter can be delivered to the nucleus in a targeted manner [26]. Moreover, although cationic nanoparticles can better accumulate in the cell nucleus [28-30], they are also easily nonspecifically absorbed by other cells and can be quickly cleared from circulation owing to their positively charged nature.

Nanoscale metal-organic frameworks (MOFs) are composed of self-assembling metal ions and organic ligands and have been widely used in the context of tumor treatment owing to their porosity and other valuable structural and chemical properties [31-36]. We have previously reported the development of a nucleustargeted MOF structure with a high photothermal conversion rate in response to strong near-infrared (NIR) light absorbance that was able to facilitate efficient tumor treatment [37]. Similar MOFs that are targeted to the nuclei and that can efficiently generate ROS may be ideal therapeutic agents to facilitate SDT tumor treatment. However, there have been relatively few reports of sonosensitizers that efficiently function in response to US irradiation [38, 39], and even fewer exhibit intrinsic nucleus-targeted activity and good biodegradability in vivo [11]. Ultra-small MOFs are considered to be highly dispersible [40] and can be efficiently metabolized in vivo in biomedical contexts [41]. These MOFs can also be utilized for intrinsic nuclear targeting owning to their ultra-small size characteristics, yet there have been few reports to date exploring this approach.

To overcome these limitations, it is thus important that a sonostable, biocompatible sonosensitizer capable of targeting to nuclei of tumor cells and generating ROS therein in an oxygen-independent manner be developed.

Herein, we report the development of an intrinsic nuclear-targeted Ti-tetrakis(4-carboxyphenyl)porphyrin (TCPP) MOF that was utilized as a sonosensitizer in an effort to overcome the limited efficacy of SDT for the treatment of orthotopic pancreatic carcinoma (Scheme 1). This novel MOF was able to be effectively internalized into cells owing to its small size $(<10 \mathrm{~nm})$ and its charge reversal property [42, 43], enabling it to be directly targeted to the nuclei and to thereby facilitate more efficient SDT. Moreover, this Ti-TCPP MOF platform was confirmed to generate ROS in a hypoxic environment, thereby facilitating oxygen-independent SDT treatment. Furthermore, our Ti-TCPP MOF exhibited good biodegradability and safety in vitro and 
in vivo. As such, we believe that this ultra-small TiTCPP MOF holds great promise for the treatment of hypoxic tumor types including pancreatic cancer.

\section{Experimental}

\section{Ti-TCPP MOF synthesis}

$\mathrm{TiCl}_{4} \cdot 2 \mathrm{THF}$ (4 mg) was dissolved in $1 \mathrm{~mL} \mathrm{~N}, \mathrm{~N}$-dimethylformamide (DMF) and combined with 5,10,15,20-tetra(pbenzoato)porphyrin $\left(\mathrm{H}^{4} \mathrm{TBP}\right)(2 \mathrm{mg})$ that had been dissolved in $2 \mathrm{~mL}$ of DMF. Next, this solution was combined with $200 \mu \mathrm{L}$ of acetic acid (AcOH). This solution was then mixed and incubated for $4 \mathrm{~h}$ at $90{ }^{\circ} \mathrm{C}$. Samples were then washed, spun down, and $10 \mathrm{mg}$ of the resultant products were combined with $5 \mathrm{~mL}$ of dimethyl sulfoxide (DMSO) and sonicated for $24 \mathrm{~h}$ in a horn sonicator (Branson Digital Sonifier SFX 550, Carouge, Switzerland) at $150 \mathrm{~W}$.

\section{ROS generation}

Singlet oxygen $\left({ }^{1} \mathrm{O}_{2}\right)$ generation was assessed with a singlet oxygen sensor green (SOSG) probe (Thermo Fisher Scientific, MA, USA) (ex/em: 504/525 nm). Superoxide $\left(\mathrm{O}_{2}{ }^{-}\right)$generation was assessed using dihydrorhodamine 123 (DHR 123, Sigma-Aldrich, USA) (ex/em: $488 / 535 \mathrm{~nm})$. Hydrogen peroxide $\left(\mathrm{H}_{2} \mathrm{O}_{2}\right)$ generation was detected at the wavelength of $560 \mathrm{~nm}$ with a hydrogen peroxide assay kit (S0038, Beyotime, China). Hydroxyl radical $(\cdot \mathrm{OH})$ generation was measured via aminophenyl fluorescein (APF) assay (Sigma-Aldrich, USA) (ex/em: 490/515 nm). Briefly, Ti-TCPP MOF was suspended in PBS at equivalent Ti concentrations of $0,10,20,40,80$, and $160 \mu \mathrm{g} \mathrm{mL}^{-1}$. These solutions were then exposed to US irradiation $\left(0.5 \mathrm{~W} \mathrm{~cm}^{-2}\right.$, duty rate $50 \%, 1 \mathrm{~min}, 1 \mathrm{MHz}$, Mettler Sonicator 740), after which fluorescence was analyzed with a multiscan spectrum (Tecan, Swiss).

\section{Assessment of Ti-TCPP MOF cellular uptake}

BxPC-3 cells were plated in 6-well $0.01 \%$ poly (Lys)coated plates $\left(1 \times 10^{5}\right.$ cells well $\left.^{-1}\right)$ overnight, after which fresh media containing $10 \mu \mathrm{g} \mathrm{mL}^{-1}$ of Ti-TCPP MOF was added for 1, 2, 6, or $8 \mathrm{~h}$. Cells were then washed three times, collected, and analyzed with a FACSCalibur flow cytometer (BD, USA).

Laser scanning confocal microscopy (LSCM) was additionally used to assess Ti-TCPP MOF uptake. For these experiments, BxPC- 3 cells $\left(1 \times 10^{5}\right)$ were grown overnight in $2 \mathrm{~mL}$ in confocal culture dishes (NETS Co., USA), after which $10 \mu \mathrm{g} \mathrm{mL}{ }^{-1}$ of Ti-TCPP MOF or PBS were added for 1,2 , or $6 \mathrm{~h}$. cells were then washed three times with PBS, fixed for 30 min with $4 \%$ formaldehyde, and stained with Hoechst 33258 Stain solution $\left(10 \mu \mathrm{g} \mathrm{mL}^{-1}\right)$ for $30 \mathrm{~min}$ prior to LSCM assessment.
Additionally, nuclear Ti levels were assessed via inductively coupled plasma optical emission spectrometry (ICP-OES) with an Optima 2100DV instrument (Perkin Elmer, USA). Mass was calculated on a per-cell basis. Briefly, BxPC-3 cells were incubated overnight and then treated by Ti-TCPP MOF $(5 \mu \mathrm{g} / \mathrm{mL}$ for Ti) for $1,2,6$ and $8 \mathrm{~h}$. After washing with PBS for three times, cells were collected, and nuclei were extracted via nucleus extraction.

\section{Cell viability assay}

Ti-TCPP MOF biocompatibility was assessed via a Cell Counting Kit-8 (CCK-8) assay (MCE, USA). Briefly, BxPC-3, Panc02, or hTERT-HPNE cells were added to 96-well plates $\left(5000\right.$ cells well $^{-1}$ ) overnight, after which media was exchanged for DMEM/1640 containing a range of Ti-TCPP MOF concentrations. Following a $24 \mathrm{~h}$ incubation, CCK-8 solution was added to each well and a microplate reader was used to assess absorbance at $450 \mathrm{~nm}$. The efficiency of SDT in vitro was also assessed by adding $100 \mu \mathrm{L}$ of BxPC-3 cells to individual wells of 96-well plates overnight, after which media containing a range of Ti-TCPP MOF concentrations was added for $6 \mathrm{~h}$. Cells were then subjected to low-intensity US treatment for $1 \mathrm{~min}\left(0.5 \mathrm{~W} \mathrm{~cm}^{-2}, 1 \mathrm{MHz}, 50 \%\right.$ duty cycle). A CCK-8 assay was then used to assess viability as above. In other experiments, BxPC-3 cells were added to 6-well plates and grown in the presence of $10 \mu \mathrm{g} \mathrm{mL}{ }^{-1}$ Ti-TCPP MOF for $6 \mathrm{~h}$. Following US irradiation for $1 \mathrm{~min}\left(0.5 \mathrm{~W} \mathrm{~cm}^{-2}\right.$, $1 \mathrm{MHz}, 50 \%$ duty cycle) and another $18 \mathrm{~h}$ incubation, cells were stained using PI and/or Annexin V-FITC, after which they were assessed via flow cytometry.

\section{In vitro DNA damage analysis}

The immunofluorescence staining of BxPC-3 cells was performed to detect DSBs. Following appropriate treatments, cells were washed and fixed in $4 \%$ paraformaldehyde for $15 \mathrm{~min}$. Cells were then stained with rabbit monoclonal anti-H2AX (1:1500) overnight at $4{ }^{\circ} \mathrm{C}$, after which they were incubated with AlexaFluor 488-conjugated anti-rabbit secondary antibody (1:400) and $2.0 \mathrm{mg} \mathrm{mL}{ }^{-1}$ DAPI for $30 \mathrm{~min}$. Cells were then imaged with a Leica fluorescence microscope $(200 \times)$.

In the DNA Ladder assay, appropriately treated BxPC-3 cells were lysed for $0.5 \mathrm{~h}$, and supernatants were collected after centrifugation. A DNA Ladder kit (Beyotime Institute of Biotechnology, China) was then used based upon provided instructions to separate DNA, which was run on a $0.8 \%$ agarose gel.

\section{Orthotopic tumor model}

Female nude mice (5-6 weeks old, BiKai Biological, Nanjing, China) were used for all animal studies, which were 
approved by the Regional Ethics Committee for Animal Experiments at The Second Affiliated Hospital of Zhejiang University School of Medicine (Permit No. 2019070). Mice were anesthetized and a $1 \mathrm{~cm}$ incision in the upper left abdominal quadrant was made. The spleen and tail of the pancreas were then exposed, and $50 \mu \mathrm{L}$ of BxPC-3 cells labeled with firefly luciferase suspended in PBS and Matrigel (phenol red-free, 2:3) were injected into the tail of the pancreas using a $0.3 \mathrm{~mm}$ needle. The spleen and pancreas were then restored to their appropriate positions within the abdomen, and the peritoneum was sutured using 4-0 absorbable sutures, after which the skin was closed with 6-0 non-absorbable sutures. Animals were then placed on a warming blanket until fully recovered from anesthetization.

\section{Pharmacokinetics and bio-distribution}

The pharmacokinetics of Ti-TCPP MOF assessed by injecting $100 \mu \mathrm{L}$ Ti-TCPP MOF $\left(10.0 \mathrm{mg} \mathrm{kg}^{-1}\right)$ into the mice through the tail vein. Blood samples were then collected at different time points $(0.17,0.5,1,2,4,8,12,18$ and $24 \mathrm{~h}$ ), lyophilized, weighed and digested with aqua regia. The Ti content in the blood was then analyzed by ICP-OES.

To evaluate the distribution of Ti-TCPP MOF in vivo, tumor-bearing mice were injected $100 \mu \mathrm{L}$ of a Ti-TCPP MOF solution (10.0 mg kg-1) or PBS (pH 7.4). Ti clearance in vivo was assessed by injecting six tumor-bearing mice with $100 \mu \mathrm{L}$ of Ti-TCPP MOF $\left(10.0 \mathrm{mg} \mathrm{kg}^{-1}\right)$. Three mice were then euthanized at baseline and three were euthanized at $8 \mathrm{~h}$ post-injection, at which time major organs and tumors were collected and Ti levels were assessed via ICP-OES analysis.

\section{In vivo fluorescence/PA imaging and therapy}

Mice were monitored until tumors had grown to 40-60 $\mathrm{mm}^{3}$ in size. After mice were injected $100 \mu \mathrm{L}$ of a Ti-

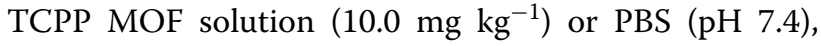
fluorescence and PA imaging (performed at $710 \mathrm{~nm}$ ) were then conducted at $0,2,4,8$, and $12 \mathrm{~h}$ post-injection. Tumor-bearing mice were randomly assigned to four treatment groups (five per group): $\mathrm{PBS}, \mathrm{PBS}+\mathrm{US}$, TiTCPP MOF, and Ti-TCPP MOF + US groups. Mice in the indicated treatment groups were injected with $100 \mu \mathrm{L}$ of PBS (pH 7.4) or $100 \mu \mathrm{L}$ PBS (pH 7.4) containing Ti-TCPP MOF $\left(10.0 \mathrm{mg} \mathrm{kg}^{-1}\right)$. At $8 \mathrm{~h}$ post-injection, US irradiation was conducted in the indicated treatment groups $(5 \mathrm{~min}$, $0.5 \mathrm{~W} \mathrm{~cm}^{-2}, 1 \mathrm{MHz}, 50 \%$ duty cycle). Then the treatment process was repeated every three days, with three treatments in total. After the intraperitoneal injection of $4 \mathrm{mg}$ of D-luciferin in $200 \mu \mathrm{L}$ of PBS, tumor sizes were assessed within 30 min using an IVIS spectrum pre-clinical in vivo imaging system. Murine survival and tumor growth were monitored for 60 days, after which major organs were collected and stained with hematoxylin and eosin (H\&E), Ki67, $\gamma$-H2AX, or tdT-mediated dUTP nick-end labeling (TUNEL) and assess via optical microscopy (DMI3000, Leica, Germany).

\section{Statistical analysis}

All experimental results were based on data from at least three independent measurements $(\mathrm{n} \geq 3)$, and all data are presented as means \pm standard deviation (SD). Graphpad Prism (version 9.0, GraphPad Software Inc.) was used for all statistical comparisons. Data were analyzed via Student's $t$-test. ${ }^{*} \mathrm{P}<0.05,{ }^{* * *} \mathrm{P}<0.01$, ${ }^{* * * *} \mathrm{P}<0.001$.

\section{Results and discussion}

Ti-TCPP MOF preparation and characterization

The approach to the preparation of our sonosensitizer (Ti-TCPP) is shown in Fig. 1a and Additional file 1: Fig. S1. Dynamic light scattering (DLS) indicated that the resultant Ti-TCPP MOF had an average diameter of $12.21 \pm 1.27 \mathrm{~nm}$ with a polydispersity index of 0.17 (Fig. 1b), and transmission electron microscopy (TEM) (Fig. 1c) revealed uniform monodispersed round Ti-TCPP MOF particles with an average diameter of $5.85 \mathrm{~nm}$. Ti-TCPP MOF particles were able to remain relatively stable for 21 days in PBS and 7 days in FBS at $4{ }^{\circ} \mathrm{C}$ (Additional file 1: Fig. S2 and S3), and for 3 days in PBS and cell culture medium at $37^{\circ} \mathrm{C}$ without any significant shifts in particle diameter (Additional file 1: Fig. S4). Under US irradiation $\left(0.5 \mathrm{~W} \mathrm{~cm}^{-2}, 1 \mathrm{MHz}, 50 \%\right.$ duty cycle, $1 \mathrm{~min}$ ), the Ti-TCPP MOF was stable, but it did exhibit an increase in size, which may be related to the catalytic reaction caused by US irradiation (Additional file 1: Fig. S5). These Ti-TCPP MOF preparations exhibited a change in zeta potential from -1.136 to $4.821 \mathrm{mV}$ upon the introduction of excess surface carboxyl groups under different $\mathrm{pH}$ conditions (Fig. 1d). Powder X-ray diffraction (XRD) measurements additionally confirmed successful Ti-TCPP MOF synthesis (Fig. 1e), the detailed crystal structure was similar to that previously published by Lan et al. [44]. XPS was additionally used to assess the elemental composition and chemical state of Ti-TCPP MOF preparations, revealing that samples contained $\mathrm{C}$, $\mathrm{O}, \mathrm{N}$, and Ti (Fig. 1f). The Ti 2p XPS spectra for these preparations exhibited two peaks at 464.58 and $458.88 \mathrm{eV}$ that were assigned to the emission from $\mathrm{Ti} 2 \mathrm{p}_{1 / 2}$ and $\mathrm{Ti}$ $2 \mathrm{p}_{3 / 2}$, respectively (Fig. $1 \mathrm{~g}$ ). Figure $1 \mathrm{~h}$ demonstrated the high-resolution N 1 s spectrum of the Ti-TCPP MOF, with two peaks at 400.58 and $398.58 \mathrm{eV}$ corresponding to $\mathrm{C}-\mathrm{N}$ and $\mathrm{C}=\mathrm{N}$, while Fig. 1i showed the high-resolution $\mathrm{C} 1 \mathrm{~s}$ spectrum for this sample. Three characteristic peaks at $288.38,286.28$, and $284.78 \mathrm{eV}$ are attributable to $\mathrm{C}=\mathrm{O}, \mathrm{C}-\mathrm{O}$, and $\mathrm{C}-\mathrm{C}$, respectively, indicating that the 


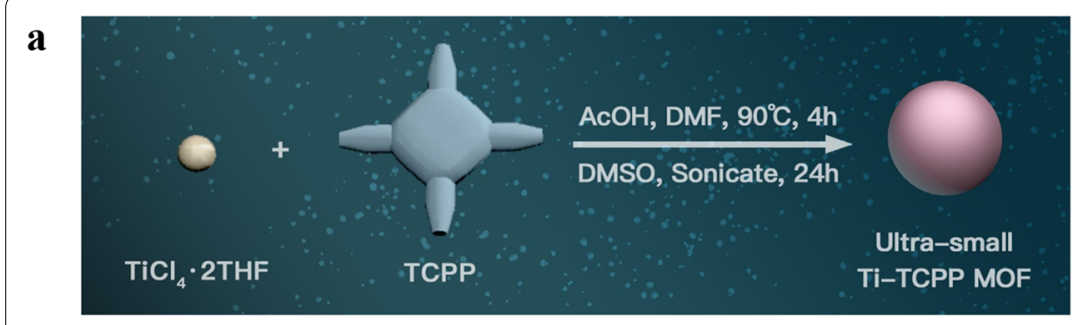

c
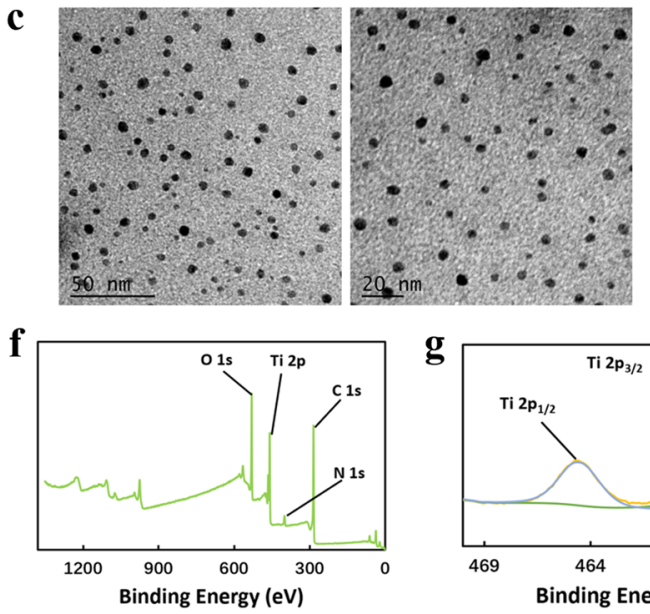

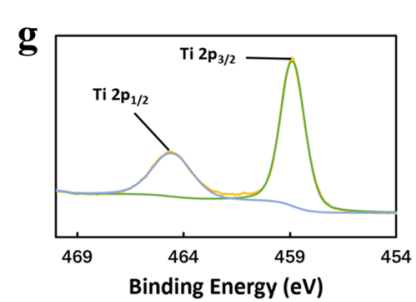

b

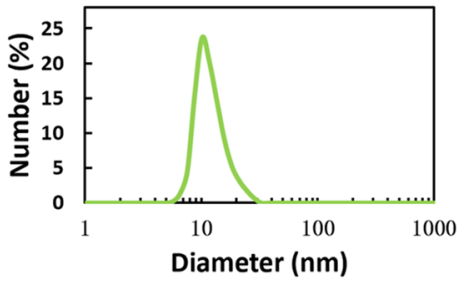

e

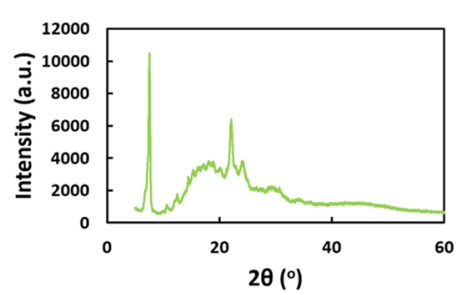

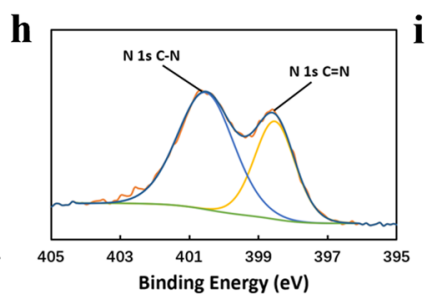

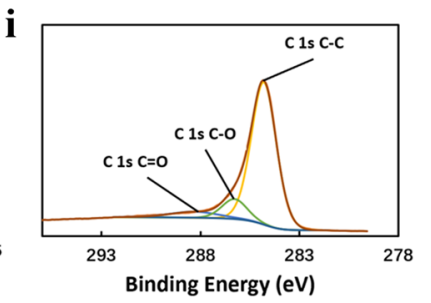

Fig. 1 a The method of ultra-small Ti-TCPP MOF synthesis. DLS curves (b), TEM images (c), zeta potentials (d), XRD pattern (e) and XPS spectra of Ti-TCPP MOF $(\mathbf{f}-\mathbf{i})$

C compound in this sample was produced using TCPP. We also detected the Brunauer-Emmett-Teller (BET) $\mathrm{N}_{2}$ adsorption/desorption of Ti-TCPP MOF (Additional file 1: Fig. S6). The $\mathrm{N}_{2}$ adsorption/desorption curve revealed type IV sorption with a surface area of $562.47 \mathrm{~m}^{2} \mathrm{~g}^{-1}$. These data confirmed that we had successfully synthesized an ultra-small Ti-TCPP MOF.

\section{Assessment of the in vitro ROS-generating efficacy and PA imaging properties of Ti-TCPP MOF}

Next, we explored the ability of Ti-TCPP MOF preparations to generate ROS. A singlet oxygen sensor green (SOSG) probe was utilized to assess ${ }^{1} \mathrm{O}_{2}$ generation following US irradiation [45], revealing an increase in ${ }^{1} \mathrm{O}_{2}$ signal in a time- and dose-dependent manner (Fig. 2a), and in a power density-dependent fashion (Additional file 1: Fig. S7a). Rapid increases in SOSG absorbance in Ti-TCPP MOF-containing solutions were consistent with robust and efficient ${ }^{1} \mathrm{O}_{2}$ generation. ROS levels produced in an oxygen-independent manner were also assessed, including $\mathrm{O}_{2}{ }^{-}$as determined with a dihydrorhodamine 123 (DHR 123) assay kit (Fig. 2b), $\mathrm{H}_{2} \mathrm{O}_{2}$ as measured with a hydrogen peroxide assay kit (Fig. $2 \mathrm{c}$ and Additional file 1: S7b), and $\cdot \mathrm{OH}$ as measured via APF assay (Fig. 2d). Upon US irradiation, characteristic absorption values consistent with $\mathrm{O}_{2}{ }^{-}, \mathrm{H}_{2} \mathrm{O}_{2}$, and $\cdot \mathrm{OH}$ generation gradually increased with Ti-TCPP MOF concentration, consistent with the utility of Ti-TCPP MOF as an effective sonosensitizer capable of simultaneously generating $\mathrm{O}_{2}^{-}, \mathrm{H}_{2} \mathrm{O}_{2}$, and $\mathrm{OH}$. The generation of these three ROS species via type I SDT was further verified by conducting these experiments in a hypoxic setting, revealing no apparent changes in $\mathrm{O}_{2}^{-}, \mathrm{H}_{2} \mathrm{O}_{2}$, or $\cdot \mathrm{OH}$ generation. Together, these findings suggested that Ti-TCPP MOF can be utilized as a promising sonosensitizer in hypoxic solid tumors such as pancreatic carcinoma.

Photoacoustic (PA) signal was first assessed in vitro under $680-900 \mathrm{~nm}$ pulse laser irradiation, revealing a robust PA signal (Additional file 1: Fig. S8). Then the PA signal at different concentrations of Ti-TCPP MOF under $710 \mathrm{~nm}$ pulse laser was calculated to confirm linearity (Fig. 2e), further supporting the promising PA properties of this MOF platform and suggesting that it can be utilized for PA imaging.

\section{Assessment of Ti-TCPP subcellular localization and antitumor activity}

Next, flow cytometry was used to explore Ti-TCPP MOF uptake by tumor cells, measuring mean fluorescence intensity (MFI) values over time. This analysis revealed a time-dependent increase in Ti-TCPP MOF uptake (Fig. 3a), with rapid increases in MFI values over the first 

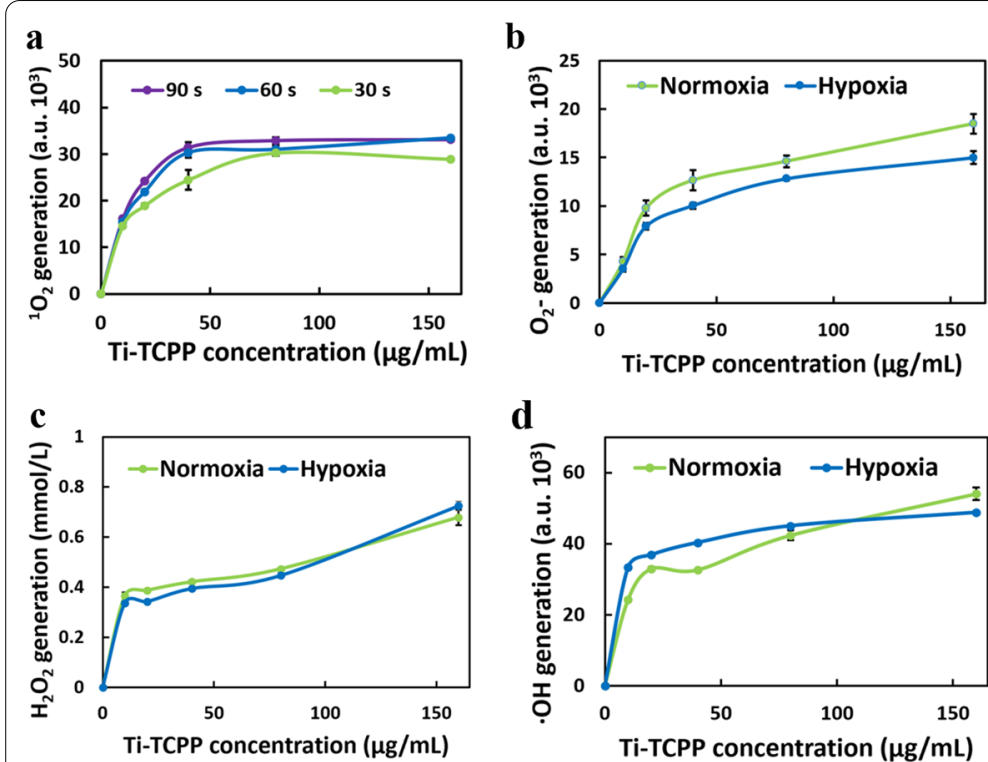

e
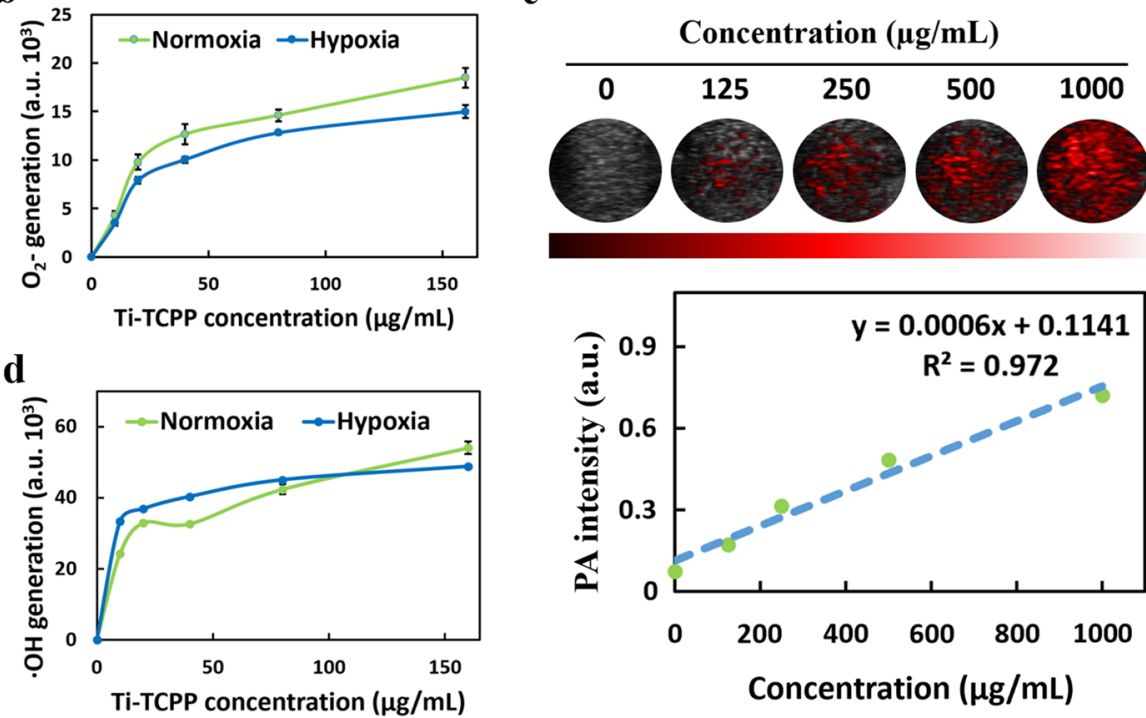

Fig. 2 Concentration-dependent ${ }^{1} \mathrm{O}_{2}$ generation (a), $\mathrm{O}_{2}{ }^{-}$generation $(\mathbf{b}), \mathrm{H}_{2} \mathrm{O}_{2}$ generation (c) and $\cdot \mathrm{OH}$ generation (d) after US irradiation under normoxic or hypoxic conditions. e Normalized intensity of photoacoustic signal versus the concentration of Ti-TCPP MOF solution

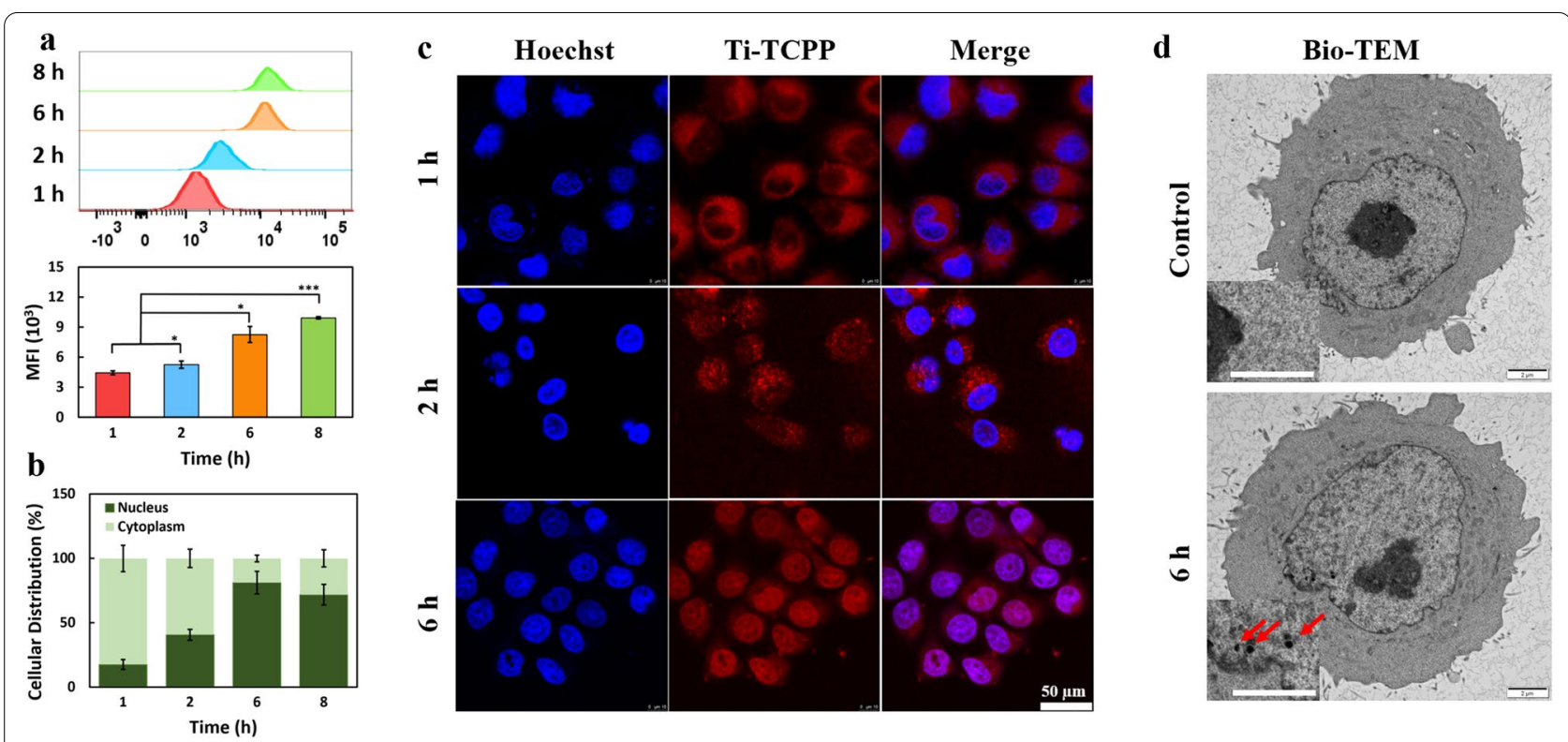

Fig. 3 Cellular uptake and nuclear localization of Ti-TCPP MOF in BXPC-3 cells. Flow cytometry (a), ICP analysis (b) and confocal images (c) of BxPC-3 cells after incubation with Ti-TCPP MOF at different time periods. Scale bar: $20 \mu \mathrm{m}$. $(\mathrm{n}=3)$. (d) Bio-TEM images of BxPC-3 cells before and $6 \mathrm{~h}$ after incubation with Ti-TCPP MOF. Scale bar: $2 \mu \mathrm{m}$. Red arrows denote Ti-TCPP MOF. ${ }^{* P}<0.05,{ }^{* * *} P<0.001$

six hours followed by slower increases over the following $2 \mathrm{~h}$. ICP-OES and LSCM were next used to assess the localization of Ti-TCPP MOF within these tumor cells. At $6 \mathrm{~h}$ post-treatment, ICP-OES analyses revealed that $81.2 \%$ of detected Ti was present in the nucleus of cells with the remaining content being present in the cytoplasm (Fig. 3b). LCSM was then conducted to confirm the nucleus-targeted activity of this MOF platform (Fig. 3c), with Hoechst being used to label BxPC-3 cell nuclei. A small quantity of Ti-TCPP MOFs was detectable in the nuclei of cells within a $2 \mathrm{~h}$ treatment period, and such nuclear accumulation rose over time before 


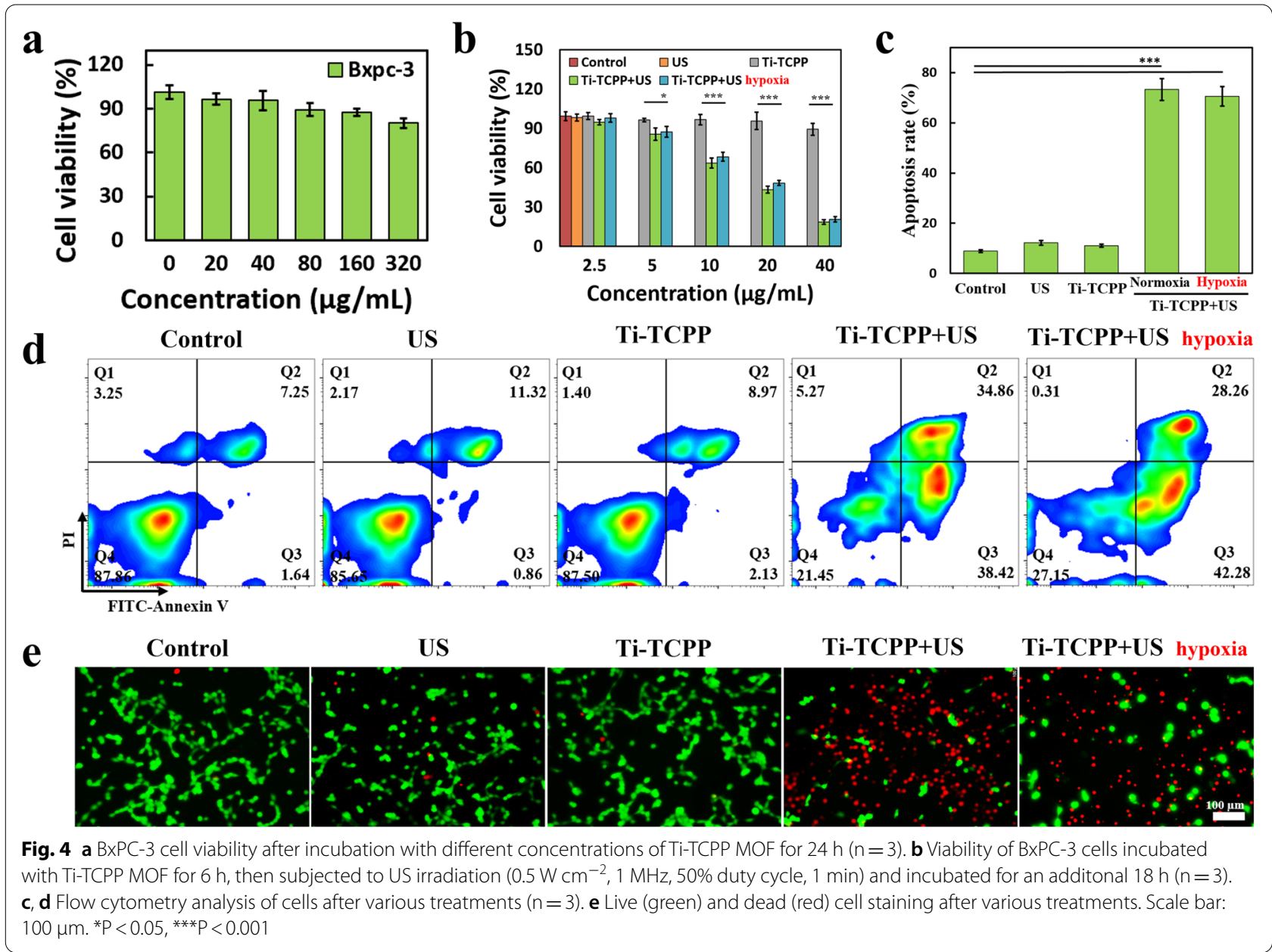

peaking at $6 \mathrm{~h}$ post-treatment, consistent with the ICPOES results. Bio-TEM examination also revealed the accumulation of Ti-TCPP MOF in the BxPC-3 cells after a $6 \mathrm{~h}$ incubation (Fig. 3d), further indicating the successful loading of Ti-TCPP MOFs. Together, these results demonstrated that this ultra-small Ti-TCPP MOF was readily internalized into the nuclei of pancreatic tumor cells. Such passive nuclear targeting may be attributable to the small particle size of this MOF and to changes in zeta potential. Exogenous nanoparticles $<9 \mathrm{~nm}$ in size have previously been reported to freely enter the nucleus through nuclear pore complexes (NPCs) [46, 47], and decreasing $\mathrm{pH}$ values can lead to a change in zeta potential values from negative to positive $[37,48]$.

A CCK-8 kit was next utilized to assess Ti-TCPP MOF biocompatibility, revealing no apparent toxicity when either tumor or normal cells (BxPC-3, Panc02, and hTERT-HPNE cell lines) after treatment for $24 \mathrm{~h}$ with a range of concentrations $(320,160,80,40$, or $20 \mu \mathrm{g} \mathrm{mL}{ }^{-1}$ ) (Fig. 4a and Additional file 1: Fig. S9). Similarly, US irradiation alone had no adverse effect on
BxPC-3 cells (Additional file 1: Fig. S10). When BxPC-3 cells were treated with Ti-TCPP MOF + US irradiation, we observed substantial ROS generation in tumor cells (Additional file 1: Fig. S11) and cell proliferation was inhibited in a dose-dependent manner at a US power of $0.5 \mathrm{~W} \mathrm{~cm}^{-2}(1 \mathrm{MHz}, 50 \%$ duty cycle, $1 \mathrm{~min})$ (Fig. $\left.4 \mathrm{~b}\right)$. At Ti-TCPP MOF concentrations above $20 \mu \mathrm{g} \mathrm{mL}{ }^{-1}$, cell survival rates fell below $50 \%$ under both normoxic and hypoxic conditions, indicating that Ti-TCPP MOFinduced SDT exhibits good therapeutic efficacy when used to kill pancreatic cancer cells in vitro. A subsequent flow cytometry analysis similarly confirmed the antitumor activity of this treatment approach, with $73.28 \%$ and $70.54 \%$ of BxPC-3 cells exhibiting apoptotic cell death following Ti-TCPP + US treatment under normoxic and hypoxic conditions, respectively (Fig. 4c, d), consistent with the results of the CCK-8 assay. A Calcein-AM/ PI dual-staining kit was also used to confirm cell viability, revealing no significant cell death in the control, TiTCPP, or US treatment groups, whereas the majority of cells in the Ti-TCPP + US group were dead (Fig. 4e). 
Together, these results confirmed the robust cytotoxicity of our nucleus-targeted SDT treatment strategy both in normoxic and hypoxic environments.

\section{Analysis of the mechanistic basis for nucleus-targeted SDT}

To understand the mechanisms underlying the efficacy of our nucleus-targeted SDT strategy, we next conducted western blotting, confocal microscopy, cell cycle progression and DNA fragmentation assays. Levels of the apoptosis-related $\mathrm{Bax}, \mathrm{Bcl}-2$, and Caspase 3 proteins were measured via Western blotting, with $\beta$-tubulin as a loading control (Fig. 5a). Following Ti-TCPP + US treatment, Caspase 3 and Bax expression increased whereas $\mathrm{Bcl}-2$ levels declined, resulting in an overall increase in the Bax/Bcl-2 ratio (Additional file 1: Fig. S12). This result was consistent with the induction of apoptotic cell death in response to treatment-induced ROS generation owing to the irreversible damage of DNA and other biomolecules in these highly proliferative cells. Apoptosis can also occur due to a disruption of the cytokinesis process [49]. Cellular proliferation depends upon cell cycle progression, with cells passing through the G0/G1, S, and
G2/M phases in sequence [50-52]. Cells that had undergone Ti-TCPP + US treatment exhibited a significant reduction in the frequency of cells in the $\mathrm{G} 2 / \mathrm{M}$ phase $(\mathrm{p}<0.05)$, and a slight increase in the number of cells in the $\mathrm{S}$ phase (39.29\%) relative to control samples (28.08\%) $(\mathrm{p}<0.05)$ (Fig. 5b). Apoptotic cell death was increasingly evident at later time points ( 24 and $48 \mathrm{~h}$ ), with respective $10.23 \%$ and $29.53 \%$ increases in the frequency of sub-G1 cell populations (Additional file 1: Fig. S13). These results indicated that nucleus-targeted SDT treatment can induce both apoptosis and cell cycle arrest at the $\mathrm{S}$ phase in tumor cells, thereby inducing mitotic catastrophe.

DNA fragmentation is a hallmark of apoptosis [53-55] and we thus utilized DNA ladder assays and confocal imaging to assess BxPC-3 cells for DNA double-strand breaks (DSBs) [56-58]. As shown in Fig. 5c and Additional file 1: Fig. S14, bright green DSB-associated $\gamma-\mathrm{H} 2 \mathrm{AX}$ foci were evident in the nuclei of cells in the Ti-TCPP + US treatment group, with Hoechst used as a nuclear counterstain. Such DNA damage was also confirmed in a DNA ladder assay (Fig. 5d), wherein Ti$\mathrm{TCPP}+\mathrm{US}$ treatment induced DNA cleavage and the

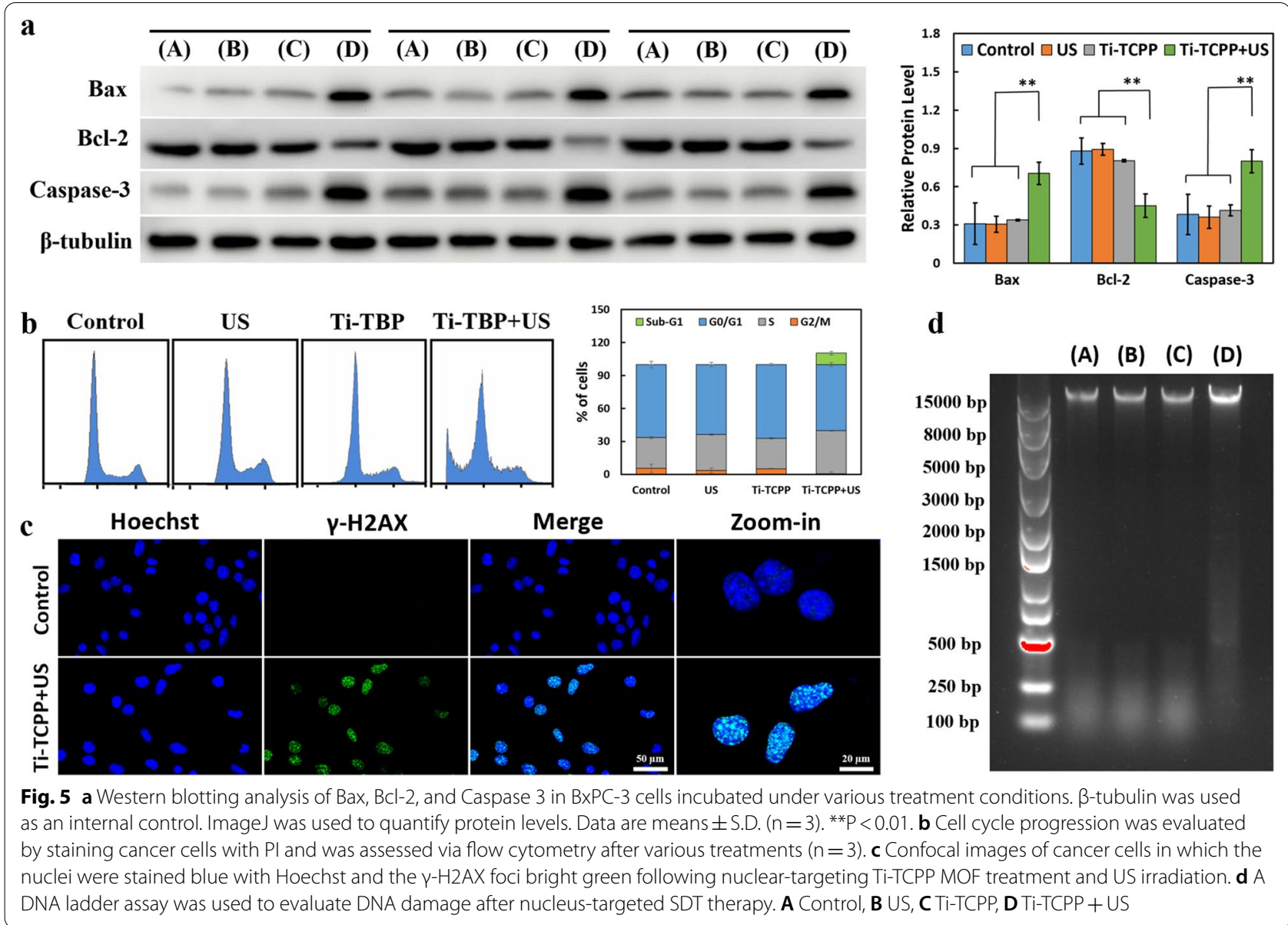


formation of a DNA fragment ladder that was absent in samples treated via US or Ti-TCPP alone. We, therefore, concluded that this combination treatment approach can induce DSB formation, likely explaining the observed cell cycle arrest and subsequent apoptotic death observed above. Both cell cycle arrest and apoptosis are effective approaches to eliminating cancer cells, highlighting the value of our nucleus-targeted SDT antitumor therapeutic strategy.

\section{In vivo biocompatibility of Ti-TCPP MOF}

We next evaluated the biosafety of our Ti-TCPP MOF platform by conducting an in vitro hemolysis assay wherein different Ti-TCPP MOF concentrations were combined with murine primary red blood cells. Relative to control samples treated with water, no apparent $\mathrm{RBC}$ lysis was observed in the other treatment groups, consistent with good biocompatibility (Fig. 6a and Additional file 1: Fig. S15). We then evaluated the in vivo toxicity of Ti-TCPP MOF preparations by injecting them into healthy nude mice, with PBS serving as a control. No significant weight loss (Additional file 1: Fig. S16) or behavioral changes were observed over 14 days following injection, nor did routine blood (Additional file 1: Fig.
S17), kidney (Fig. 6b), liver function analyses (Fig. 6c) or H\&E staining of primary organs reveal any treatmentrelated changes relative to control animals (Fig. 6d). Together these data indicated that Ti-TCPP MOF exhibits a high degree of biosafety and will not cause significant treatment-related toxicity.

\section{Assessment of Ti-TCPP MOF in vivo distribution and pharmacokinetics}

To more reliably assess the intratumoral accumulation of Ti-TCPP MOF in vivo, fluorescent and PA imaging were conducted at a range of time points $(0,2,4,8$, and $12 \mathrm{~h})$ following intravenous injection into mice bearing $\mathrm{BxPC}-3$ tumors. Fluorescence increased in a time-dependent manner, reaching maximal fluorescence at $8 \mathrm{~h}$ post-injection and with a strong signal remaining evident at the $12 \mathrm{~h}$ time point (Fig. 7a and Additional file 1: Fig. S18a). Analyses of primary organs from these mice at $12 \mathrm{~h}$ postinjection further supported the accumulation of Ti-TCPP MOF within tumors (Additional file 1: Fig. S18b). Analyses of the in vivo PA signal yielded comparable results to those of fluorescence intensity analyses (Fig. 7b).

Next, ICP-OES was used to measure Ti concentrations in the blood and major organs of these mice, revealing a

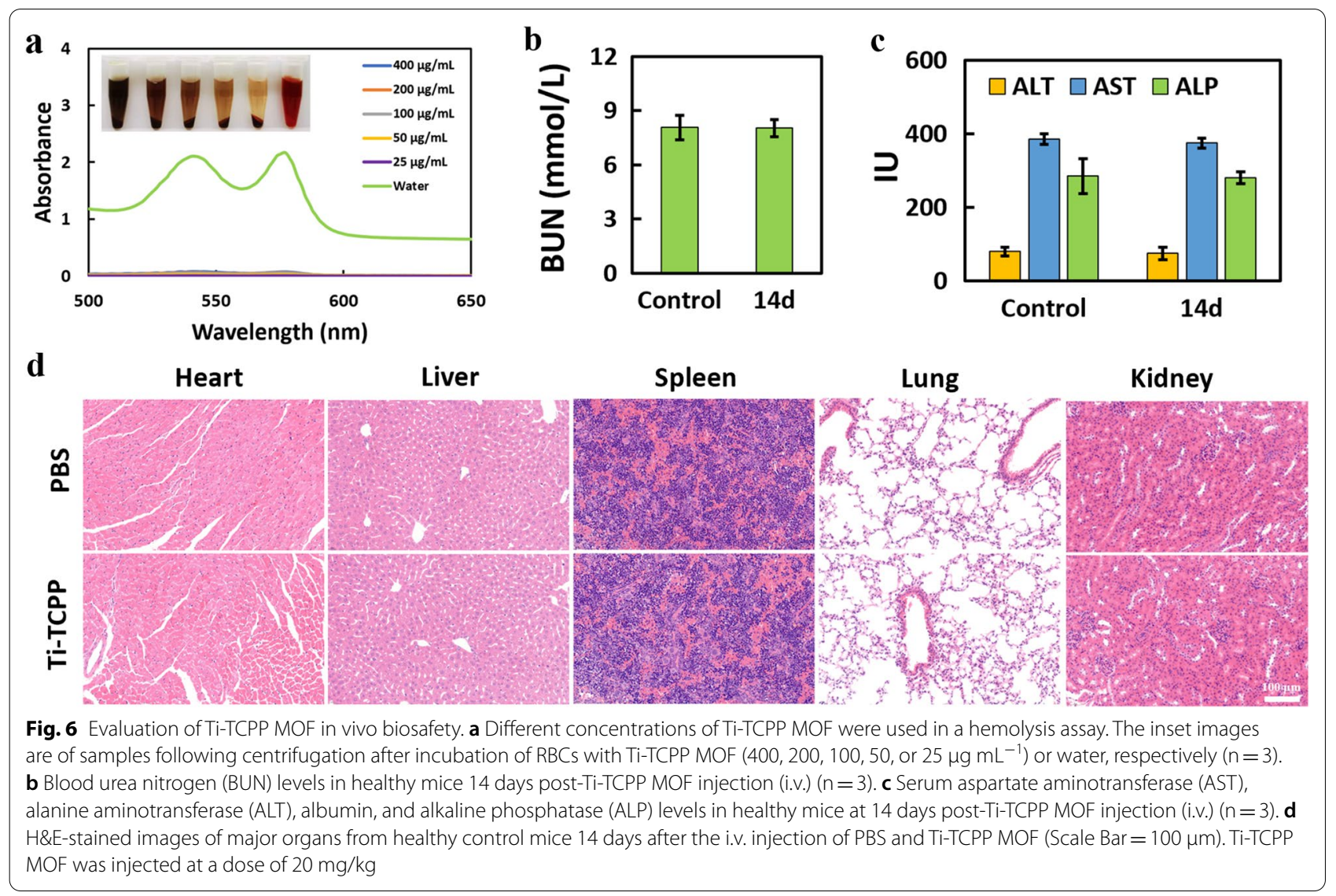




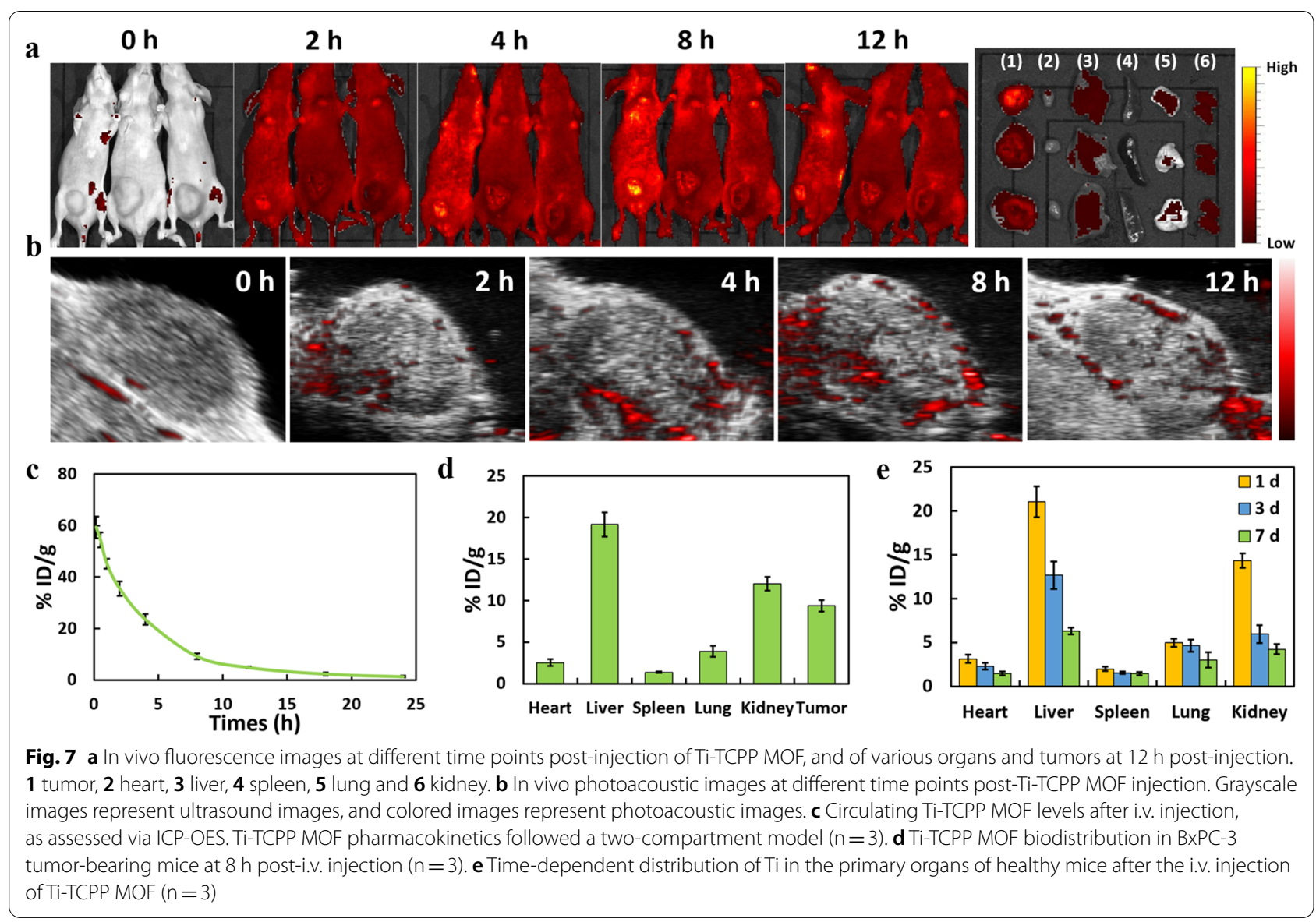

gradual reduction in Ti-TCPP MOF concentrations in the blood within $24 \mathrm{~h}$ with a half-life of $5.09 \pm 0.38 \mathrm{~h}$ calculated using a two-compartment model (Fig. 7c). This relatively long half-life facilitated the passive accumulation of Ti-TCPP MOF within tumors owing to the enhanced permeability and retention effect, with an accumulation of $9.37 \pm 0.68 \% \mathrm{ID} / \mathrm{g}$ at $8 \mathrm{~h}$ post-injection within murine tumors (Fig. $7 \mathrm{~d}$ ). Ti levels within major organs were assessed over a 7-day period following treatment, revealing relatively low concentrations within 3 days, and with nearly complete clearance after one week (Fig. 7e). These results suggest that while ultra-small Ti-TCPP MOF can efficiently accumulate within tumors, it can be readily metabolized by the liver and kidney, thus reducing its overall accumulation in the body, and facilitating excellent biocompatibility and safety.

\section{In vivo antitumor efficacy}

After establishing an orthotopic model of murine pancreatic cancer, mice were randomly assigned to four treatment groups, with three total treatments, and tumor weight, body weight and fluorescence images being captured every three days (Fig. 8a). A $2.0 \mathrm{~cm}$-thick US gel pad was utilized to reduce thermal effects during US irradiation.

Mice treated with Ti-TCPP MOF+US exhibited significant inhibition of tumor growth, whereas tumor-associated fluorescent signal rapidly increased in intensity over time in the other three treatment groups (Fig. 8b, c). Mice in the Ti-TCPP + US group survived for over 60 days, whereas mice in the other three groups survived a maximum of 34 days (Fig. $8 d$ ). These results were consistent with the efficacy of our nucleus-targeted SDT approach. Importantly, no significant decreases in murine body weight were observed over the 15-day treatment period (Additional file 1: Fig. S19), nor were any significant changes observed upon histopathological examination of H\&E-stained heart, liver, spleen, lung, and kidney tissue samples from the mice in any treatment group, consistent with the safety of this therapeutic strategy (Additional file 1: Fig. S20). Tumors were then collected and subjected to $\mathrm{H} \& \mathrm{E}$, TUNEL, Ki67, and $\gamma-\mathrm{H} 2 \mathrm{AX}$ staining to evaluate the efficacy of this SDT treatment approach (Fig. 8e). H\&E-stained tumor sections revealed clear nuclear fragmentation and a reduction in nucleus size 

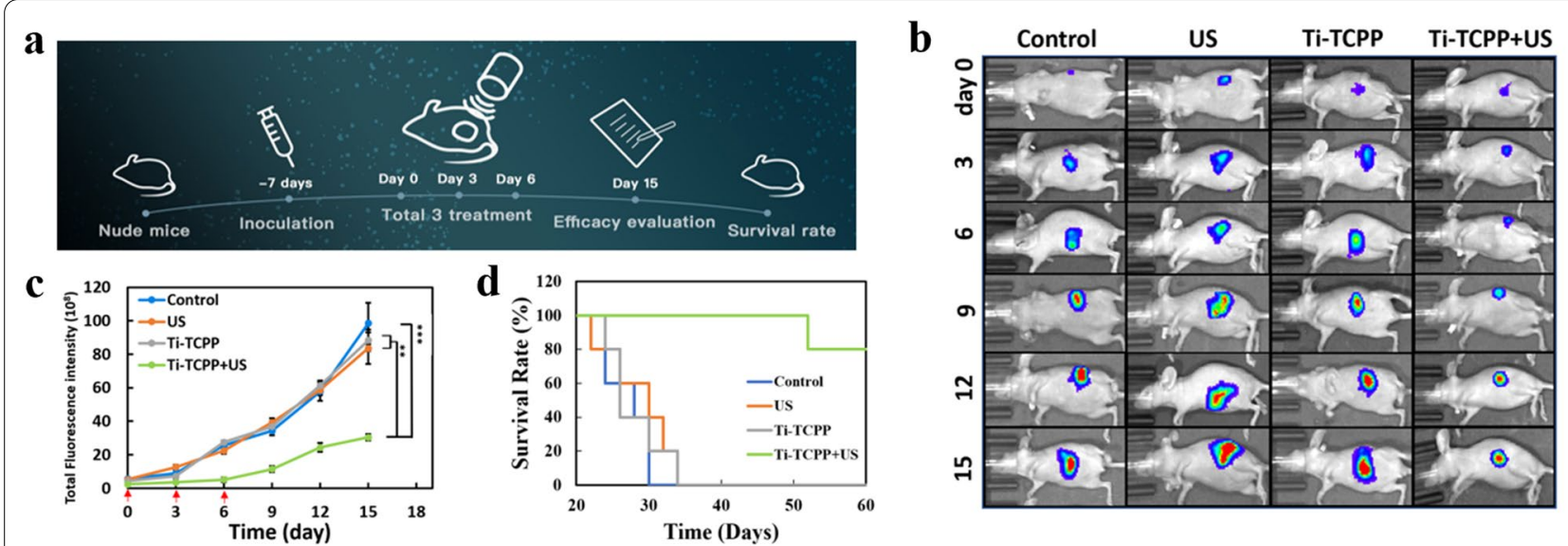

e
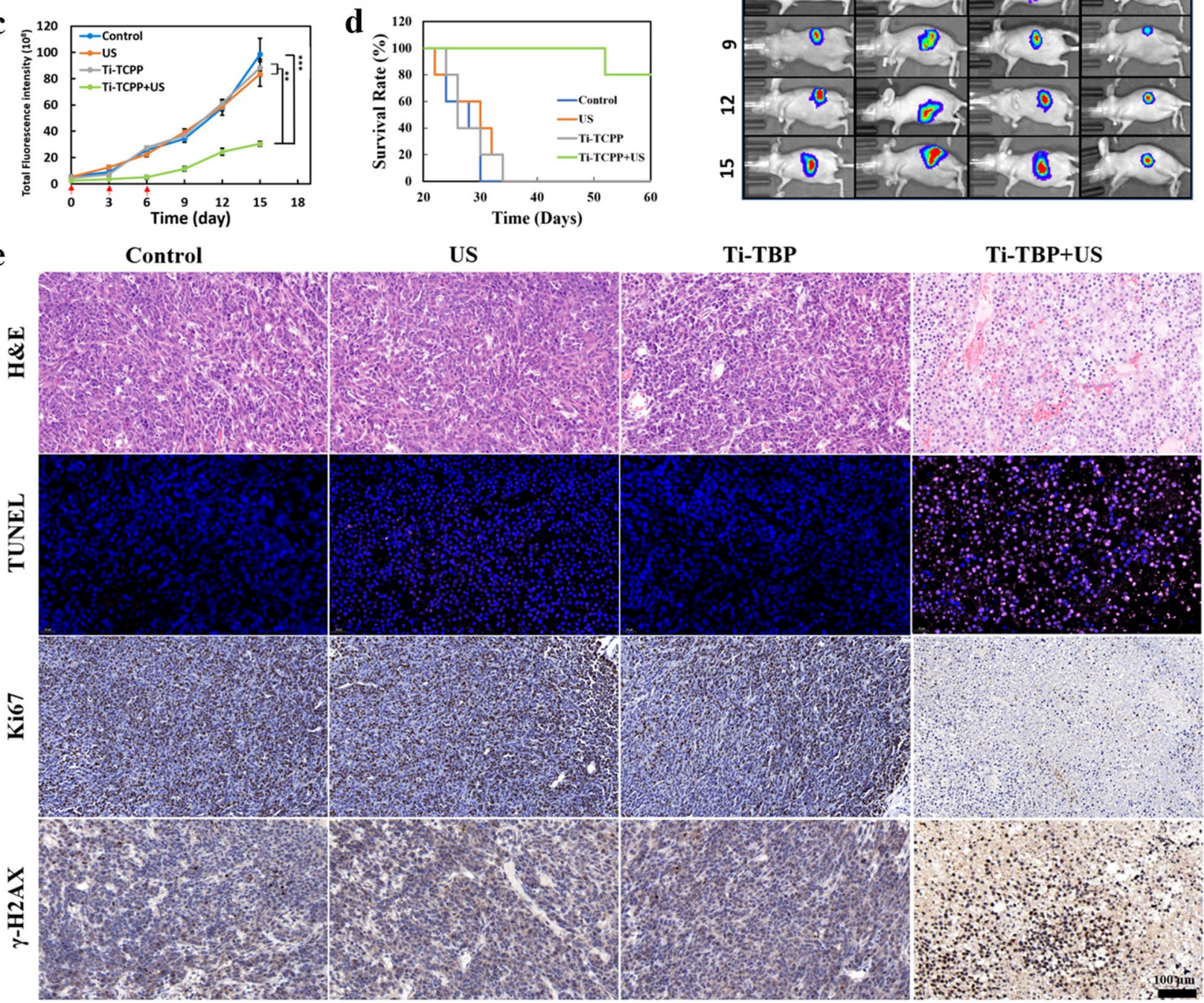

Fig. 8 In vivo antitumor evaluation. a Schematic illustration of the therapy process. b Fluorescence images of tumor-bearing mice after various treatments. c Tumor fluorescence intensity changes and $\mathbf{d}$ survival rates of tumor-bearing mice after different treatments. Data are means \pm SD $(n=5)$. e H\&E, TUNEL, Ki67, and $\gamma-H 2 A X$ staining of tumors 15 day after various treatments (Scale bar $=100 \mu m$ )

in cells in the Ti-TCPP MOF + US group. The TUNEL assay has been widely used to detect apoptotic cells in tumor tissues while Ki67 is a sensitive biomarker for cell proliferation $[59,60]$. Consistent with H\&E staining results, TUNEL staining confirmed that this treatment combination greatly increased the rate of tumor cell apoptosis, while Ki67 staining indicated a reduction in tumor cell proliferation following Ti-TCPP MOF + US treatment relative to other treatments. Intranuclear $\gamma$-H2AX levels were also increased in tumor cells from mice treated with Ti-TCPP MOF + US, consistent with severe DNA damage. Overall, these results thus confirmed the direct antitumor activity of Ti-TCPP MOF as a nucleus-targeted sonosensitizing agent. 


\section{Conclusions}

In conclusion, we developed an ultra-small Ti-TCPP MOF for effective nucleus-targeted SDT. These preparations were able to intrinsically target cellular nuclei without additional modification, therein destroying DNA, inducing $\mathrm{S}$ phase cell cycle arrest, and thereby driving apoptotic cell death. This MOF platform was able to efficiently generate ROS, thereby facilitating both type I and type II SDT in an oxygen-independent manner. This activity was consistent with a promising approach to the treatment of hypoxic solid tumors. In in vivo experiments, Ti-TCPP MOF was almost fully cleared within 7 days of treatment without any evidence of hematological or histopathological toxicity. As such, our nucleustargeted Ti-TCPP MOF platform represents a novel and efficient approach to facilitating SDT in response to lowintensity ultrasound.

\begin{abstract}
Abbreviations
MOF: Metal-organic framework; ROS: Reactive oxygen species; NIR: Nearinfrared; DMF: N,N-dimethylformamide; $\mathrm{H}_{4}$ TBP: 5,10,15,20-Tetra(p-benzoato) porphyrin; $\mathrm{AcOH}$ : Acetic acid; DMSO: Dimethyl sulfoxide; ${ }^{1} \mathrm{O}_{2}$ : Singlet oxygen; SOSG: Singlet oxygen sensor green; $\mathrm{O}^{-}$: Superoxide; $\mathrm{H}_{2} \mathrm{O}_{2}$ : Hydrogen peroxide; $\cdot \mathrm{OH}$ : Hydroxyl radical; APF: Aminophenyl fluorescein; CLSM: Confocal laser scanning microscopy; ICP-OES: Inductively coupled plasma optical emission spectrometry; CCK-8: Cell counting kit-8; DHR 123: Dihydrorhodamine 123; PA: Photoacoustic; MFI: Mean fluorescence intensity; NPCs: Nuclear pore complexes; DSBs: DNA double-strand breaks.
\end{abstract}

\section{Supplementary Information}

The online version contains supplementary material available at https://doi. org/10.1186/s12951-021-01060-7.

Additional file 1: Fig. S1. Structural formula and synthesis method of ultra-small Ti-TCPP MOF. Fig. S2. TEM images and DLS measurement of Ti-TCPP MOF in PBS for 21 days (a) and FBS for 7 days (b) at $4{ }^{\circ} \mathrm{C}$. Scale bar: $50 \mathrm{~nm}$. Fig. S3. XRD pattern of Ti-TCPP MOF in PBS for 21 days at $4{ }^{\circ} \mathrm{C}$. Fig. S4. TEM images and DLS measurement of Ti-TCPP MOF in PBS (a) and FBS for 3 days (b) at $37^{\circ} \mathrm{C}$. Scale bar: $50 \mathrm{~nm}$. Fig. S5. TEM images and DLS measurement of Ti-TCPP MOF in PBS after US irradiation $\left(0.5 \mathrm{~W} \mathrm{~cm}{ }^{-2}\right.$, $1 \mathrm{MHz}, 50 \%$ duty cycle, $1 \mathrm{~min}$ ). Scale bar: $50 \mathrm{~nm}$. Fig. S6. $\mathrm{N}_{2}$ adsorption/ desorption result of Ti-TCPP MOF. Fig. S7. (a) Power-dependent ${ }^{1} \mathrm{O}_{2}$ generation after US irradiation under normoxia conditions. (b) A standard curve of $\mathrm{H}_{2} \mathrm{O}_{2}$ generation measured by multiscan spectrum. Fig. S8. The PA signal of Ti-TCPP MOF under 680-900 nm pulse laser irradiation in vitro. Fig. S9. Cell viability of Panc02 (a) and hTERT-HPNE (b) cells after incubated with Ti-TCPP MOF for 24 h. Fig. S10. Cell viability of BXPC-3 cells after US irradiation (1 MHz, 50\% duty cycle, $1 \mathrm{~min}$ ) for 24 h. Fig. S11. Detection of ROS generation by DCFH-DA kit in tumor cells. Fig. S12. The quantified levels of $\mathrm{Bax} / \mathrm{Bcl}-2$ was analyzed by Image J software. The data were expressed as mean \pm S.D. $(n=3) .{ }^{* *} P<0.001$. Fig. $\mathbf{S 1 3}$. Cell cycle analysis determined by flow cytometry (a) and statistical analysis of sub-G1 phase (b) after treatment with Ti-TCPP + US at different time period $(n=3)$. ${ }^{* * *} p<0.001$. Fig. S14. Confocal images of cancer cells in which the nuclei were stained blue with Hoechst and the $\mathrm{\gamma}$-H2AX foci bright green following nuclear-targeting Ti-TCPP MOF treatment or US irradiation. Fig. S15. Hemolysis coefficient after incubation of RBCs with Ti-TCPP MOF (400, 200, 100,50 , or $\left.25 \mu \mathrm{g} \mathrm{mL}^{-1}\right)$ or water, respectively $(n=3)$. The hemolysis coefficient of Ti-TCPP MOF was less than $5 \%$, indicating the good biocompatibility of Ti-TCPP MOF. Fig. S16. The body weight changes of healthy mice treated by PBS or Ti-TCPP MOF during 14 days. Fig. S17. Blood routine analysis. Blood levels of WBC, RBC, HGB, HCT, MCHC, PLT, MCV and MCH of health mice after $14 \mathrm{~d}$ post injection (i.v.) of Ti-TCPP MOF, PBS was set as control. Fig. S18. (a) Statistics analysis of tumor fluorescence intensity after i.v. Ti-TCPP MOF injection at different time period $(n=3)$. (b) Statistics analysis of fluorescence intensity of main organs $12 \mathrm{~h}$ after i.v. Ti-TCPP MOF injection $(n=3)$. Fig. S19. The body weight of mice after various treat-

ment for 15 days, indicating no acute toxicity to mice major organs. $n=5$.

Fig. S20. Histological analysis of the main organs (heart, liver, spleen, lung and kidney) of un-treated mice (control) and mice treated with US, Ti-TCPP MOF and Ti-TCPP MOF + US for 15 days. Scale bar $=100 \mu \mathrm{m}$.

\section{Acknowledgements}

Not applicable.

\section{Authors' contributions}

$\mathrm{PH}$ and $\mathrm{ZJ}$ provided financial support and participated in the supervision and coordination of the study; TZ and ZJ conceived and designed the experiments; TZ, YS, JC, CZ, and JW performed most of the experiments; TZ and ZJ analyzed the data; $\mathrm{PH}, \mathrm{ZJ}$ and TZ contributed to the writing, review and revision of the manuscript. All authors read and approved the final manuscript.

\section{Funding}

This work was supported by National Natural Science Foundation of China (82030048, 81901871, 32101153), Key Research and Development Program of Zhejiang Province (2019C03077), Natural Science Foundation of Zhejiang Province (LQ19H180004, LGF19C100001, LQ20H180009), China Postdoctoral Science Foundation (2020M680395).

\section{Declarations}

\section{Ethics approval and consent to participate}

All animal experiments were conducted under the Regional Ethics Committee for Animal Experiments at The Second Affiliated Hospital of Zhejiang University School of Medicine, China (Permit No. 2019-070).

\section{Consent for publication}

All authors agreed to submit this manuscript.

\section{Competing interests}

The authors declare no competing financial interest.

\section{Author details}

${ }^{1}$ Department of Ultrasound in Medicine, The Second Affiliated Hospital of Zhejiang University School of Medicine, No.88 Jiefang Road, Shangcheng District, Hangzhou 310009, People's Republic of China. ${ }^{2}$ Research Center of Ultrasound in Medicine and Biomedical Engineering, The Second Affiliated Hospital of Zhejiang University School of Medicine, No.88 Jiefang Road, Shangcheng District, Hangzhou 310009, People's Republic of China. ${ }^{3}$ Institute of Engineering Medicine, Beijing Institute of Technology, No. 5, South Street, Zhongguancun, Haidian District, Beijing 100081, People's Republic of China.

Received: 11 June 2021 Accepted: 26 September 2021

Published online: 12 October 2021

\section{References}

1. Pan X, Wang W, Huang Z, et al. MOF-derived double-layer hollow nanoparticles with oxygen generation for multimodal imaging-guided sonodynamic therapy. Angew Chem Int Ed Engl. 2020. https://doi.org/ 10.1002/anie.202004894.

2. Zhang Y, Bi L, Hu Z, et al. Hematoporphyrin monomethyl ether-mediated sonodynamic therapy induces A-253 cell apoptosis. Oncol Lett. 2020;19(4):3223-8.

3. Chang N, Qin D, Wu P, et al. IR780 loaded perfluorohexane nanodroplets for efficient sonodynamic effect induced by short-pulsed focused ultrasound. Ultrason Sonochem. 2019;53:59-67. 
4. Ma A, Chen H, Cui Y, et al. Metalloporphyrin complex-based nanosonosensitizers for deep-tissue tumor theranostics by noninvasive sonodynamic therapy. Small. 2019;15(5): e1804028.

5. Lafond M, Yoshizawa S, Umemura SI. Sonodynamic therapy: advances and challenges in clinical translation. J Ultrasound Med. 2019;38(3):567-80.

6. Prescott M, Mitchell J, Totti S, et al. Sonodynamic therapy combined with novel anti-cancer agents, sanguinarine and ginger root extract: synergistic increase in toxicity in the presence of PANC-1 cells in vitro. Ultrason Sonochem. 2018;40(Pt B):72-80.

7. Lu K, He C, Guo N, et al. Chlorin-based nanoscale metal-organic framework systemically rejects colorectal cancers via synergistic photodynamic therapy and checkpoint blockade immunotherapy. J Am Chem Soc. 2016;138(38):12502-10.

8. Park J, Jiang Q, Feng D, et al. Size-controlled synthesis of porphyrinic metal-organic framework and functionalization for targeted photodynamic therapy. J Am Chem Soc. 2016;138(10):3518-25.

9. Brown JM, Wilson WR. Exploiting tumour hypoxia in cancer treatment. Nat Rev Cancer. 2004;4(6):437-47.

10. Zhao H, Zhao B, Li L, et al. Biomimetic decoy inhibits tumor growth and lung metastasis by reversing the drawbacks of sonodynamic therapy. Adv Healthc Mater. 2020;9(1): e1901335.

11. Zhu P, Chen YShi J, . Nanoenzyme-augmented cancer sonodynamic therapy by catalytic tumor oxygenation. ACS Nano. 2018;12(4):3780-95.

12. Koong AC, Mehta VK, Le QT, et al. Pancreatic tumors show high levels of hypoxia. Int J Radiat Oncol Biol Phys. 2000;48(4):919-22.

13. Li E, Sun Y, Lv G, et al. Sinoporphyrin sodium based sonodynamic therapy induces anti-tumor effects in hepatocellular carcinoma and activates p53/caspase 3 axis. Int J Biochem Cell Biol. 2019;113:104-14.

14. Vaupel $P$, Hockel M, Mayer A. Detection and characterization of tumor hypoxia using pO2 histography. Antioxid Redox Signal. 2007;9(8):1221-35.

15. Li $X$, Peng $X H$, Zheng BD, et al. New application of phthalocyanine molecules: from photodynamic therapy to photothermal therapy by means of structural regulation rather than formation of aggregates. Chem Sci. 2018;9(8):2098-104.

16. Juarranz A, Jaen P, Sanz-Rodriguez F, et al. Photodynamic therapy of cancer. Basic principles and applications. Clin Transl Oncol. 2008;10(3):148-54.

17. Rengeng L, Qianyu Z, Yuehong L, et al. Sonodynamic therapy, a treatment developing from photodynamic therapy. Photodiagnosis Photodyn Ther. 2017;19:159-66.

18. Nesbitt H, Sheng Y, Kamila S, et al. Gemcitabine loaded microbubbles for targeted chemo-sonodynamic therapy of pancreatic cancer. J Control Release. 2018;279:8-16.

19. Chen Y, Du M, Yu J, et al. Nanobiohybrids: a synergistic integration of bacteria and nanomaterials in cancer therapy. BIO Integration. 2020;1(1):25-36.

20. Pan L, Liu JShi J, . Cancer cell nucleus-targeting nanocomposites for advanced tumor therapeutics. Chem Soc Rev. 2018;47(18):6930-46.

21. Cheng H, Fan JH, Zhao LP, et al. Chimeric peptide engineered exosomes for dual-stage light guided plasma membrane and nucleus targeted photodynamic therapy. Biomaterials. 2019:211:14-24.

22. Liu Z, Qiu K, Liao X, et al. Nucleus-targeting ultrasmall ruthenium(iv) oxide nanoparticles for photoacoustic imaging and low-temperature photothermal therapy in the NIR-II window. Chem Commun. 2020;56(20):3019-22.

23. Zhang $\mathrm{P}$, Huang $\mathrm{H}$, Banerjee $\mathrm{S}$, et al. Nucleus-targeted organoiridiumalbumin conjugate for photodynamic cancer therapy. Angew Chem Int Ed. 2019;58(8):2350-4

24. Ma X, Gong N, Zhong L, et al. Future of nanotherapeutics: targeting the cellular sub-organelles. Biomaterials. 2016;97:10-21.

25. Gao X, Zhang J, Huang Z, et al. Reducing interstitial fluid pressure and inhibiting pulmonary metastasis of breast cancer by gelatin modified cationic lipid nanoparticles. ACS Appl Mater Interfaces. 2017;9(35):29457-68.

26. Pan L, He Q, Liu J, et al. Nuclear-targeted drug delivery of TAT peptideconjugated monodisperse mesoporous silica nanoparticles. J Am Chem Soc. 2012;134(13):5722-5.

27. Li Y, Mei T, Han S, et al. Cathepsin B-responsive nanodrug delivery systems for precise diagnosis and targeted therapy of malignant tumors. Chin Chem Lett. 2020;31(12):3027-40.
28. Cao Y, Wu T, Zhang K, et al. Engineered exosome-mediated near-infraredII region $\mathrm{V} 2 \mathrm{C}$ quantum dot delivery for nucleus-target low-temperature photothermal therapy. ACS Nano. 2019;13(2):1499-510.

29. Zhang C, Jin R, Zhao P, et al. A family of cationic polyamides for in vitro and in vivo gene transfection. Acta Biomater. 2015;22:120-30.

30. Vaidyanathan S, Chen J, Orr BG, et al. Cationic polymer intercalation into the lipid membrane enables intact polyplex DNA escape from endosomes for gene delivery. Mol Pharm. 2016;13(6):1967-78.

31. Zhang T, Jiang $Z$, Chen $L$, et al. PCN-Fe(III)-PTX nanoparticles for MRI guided high efficiency chemo-photodynamic therapy in pancreatic cancer through alleviating tumor hypoxia. Nano Res. 2020;13(1):273-81.

32. Pan X, Bai L, Wang H, et al. Metal-organic-framework-derived carbon nanostructure augmented sonodynamic cancer therapy. Adv Mater. 2018;30(23): e1800180.

33. Zhang K, Meng XD, Cao Y, et al. Metal-organic framework nanoshuttle for synergistic photodynamic and low-temperature photothermal therapy. Adv Funct Mater. 2018. https://doi.org/10.1002/adfm.201804634.

34. Horcajada P, Gref R, Baati T, et al. Metal-organic frameworks in biomedicine. Chem Rev. 2012;112(2):1232-68.

35. Chen WH, Liao WC, Sohn YS, et al. Stimuli-responsive nucleic acid-based polyacrylamide hydrogel-coated metal-organic framework nanoparticles for controlled drug release. Adv Funct Mater. 2018;28(8):1705137.

36. Chen WH, Luo GF, Zhang XZ. Recent advances in subcellular targeted cancer therapy based on functional materials. Adv Mater. 2019;31(3):1802725.

37. Jiang Z, Yuan B, Wang Y, et al. Near-infrared heptamethine cyanine dyebased nanoscale coordination polymers with intrinsic nucleus-targeting for low temperature photothermal therapy. Nano Today. 2020. https:// doi.org/10.1016/j.nantod.2020.100910.

38. Wang $D, W u H, L i m ~ W Q$, et al. A mesoporous nanoenzyme derived from metal-organic frameworks with endogenous oxygen generation to alleviate tumor hypoxia for significantly enhanced photodynamic therapy. Adv Mater. 2019;31(27): e1901893.

39. Zhang A, Pan S, Zhang Y, et al. Carbon-gold hybrid nanoprobes for realtime imaging, photothermal/photodynamic and nanozyme oxidative therapy. Theranostics. 2019;9(12):3443-58.

40. Xu X. Controllable synthesis of ultra-small metal-organic framework nanocrystals composed of copper (ii) carboxylates. Nanoscale. 2016;8(37):16725-32.

41. Wang H, Yu D, Fang J, et al. Renal-clearable porphyrinic metal-organic framework nanodots for enhanced photodynamic therapy. ACS Nano. 2019;13(8):9206-17.

42. Gao G, Jiang YW, Sun W, et al. Molecular targeting-mediated mild-temperature photothermal therapy with a smart albumin-based nanodrug. Small. 2019;15(33): e1900501.

43. Liu L-H, Qiu W-X, Zhang Y-H, et al. A charge reversible self-delivery chimeric peptide with cell membrane-targeting properties for enhanced photodynamic therapy. Adv Funct Mater. 2017. https://doi.org/10.1002/ adfm.201700220.

44. Lan G, Ni K, Veroneau SS, et al. Titanium-based nanoscale metalorganic framework for type I photodynamic therapy. J Am Chem Soc. 2019;141(10):4204-8

45. Yang Z, Wang J, Ai S, et al. Self-generating oxygen enhanced mitochondrion-targeted photodynamic therapy for tumor treatment with hypoxia scavenging. Theranostics. 2019:9(23):6809-23.

46. Paine PL, Moore LC, Horowitz SB. Nuclear envelope permeability. Nature. 1975;254(5496):109-14

47. Yuan Y, Liu C, Qian J, et al. Size-mediated cytotoxicity and apoptosis of hydroxyapatite nanoparticles in human hepatoma HepG2 cells. Biomaterials. 2010;31(4):730-40.

48. Zhang M, Chen X, Li C, et al. Charge-reversal nanocarriers: an emerging paradigm for smart cancer nanomedicine. J Control Release. 2020;319:46-62.

49. Kang B, Mackey MAEl-Sayed MA, . Nuclear targeting of gold nanoparticles in cancer cells induces DNA damage, causing cytokinesis arrest and apoptosis. J Am Chem Soc. 2010;132(5):1517-9.

50. Zhong Z, Zhou F, Wang D, et al. Expression of KLF9 in pancreatic cancer and its effects on the invasion, migration, apoptosis, cell cycle distribution, and proliferation of pancreatic cancer cell lines. Oncol Rep. 2018:40(6):3852-60. 
51. Azorsa DO, Gonzales IM, Basu GD, et al. Synthetic lethal RNAi screening identifies sensitizing targets for gemcitabine therapy in pancreatic cancer. J Transl Med. 2009;7:43.

52. Xu H, Cheung IY, Wei XX, et al. Checkpoint kinase inhibitor synergizes with DNA-damaging agents in G1 checkpoint-defective neuroblastoma. Int J Cancer. 2011;129(8):1953-62.

53. Majtnerova P, Rousar T. An overview of apoptosis assays detecting DNA fragmentation. Mol Biol Rep. 2018;45(5):1469-78.

54. Zhou J, Wang Q, Geng S, et al. Construction and evaluation of tumor nucleus-targeting nanocomposite for cancer dual-mode imaging-guiding photodynamic therapy/photothermal therapy. Mater Sci Eng C Mater Biol Appl. 2019;102:541-51.

55. Yumita $N$, Iwase $Y$, Nishi $K$, et al. Involvement of reactive oxygen species in sonodynamically induced apoptosis using a novel porphyrin derivative. Theranostics. 2012;2(9):880-8.

56. Luan S, Yun X, Rao W, et al. Emamectin benzoate induces ROS-mediated DNA damage and apoptosis in Trichoplusia Tn5B1-4 cells. Chem Biol Interact. 2017;273:90-8.

57. Liu S, Cao W, Yu L, et al. Zinc(II) complexes containing bis-benzimidazole derivatives as a new class of apoptosis inducers that trigger DNA damage-mediated p53 phosphorylation in cancer cells. Dalton Trans. 2013:42(16):5932-40.

58. Bian C, Zhang C, Luo T, et al. NADP(+) is an endogenous PARP inhibitor in DNA damage response and tumor suppression. Nat Commun. 2019;10(1):693.

59. Yan X, Yang L, Feng G, et al. Lup-20(29)-en-3beta,28-di-yl-nitrooxy acetate affects MCF-7 proliferation through the crosstalk between apoptosis and autophagy in mitochondria. Cell Death Dis. 2018;9(2):241.

60. Wu J, Bremner DH, Niu S, et al. Chemodrug-gated biodegradable hollow mesoporous organosilica nanotheranostics for multimodal imagingguided low-temperature photothermal therapy/chemotherapy of cancer. ACS Appl Mater Interfaces. 2018;10(49):42115-26.

\section{Publisher's Note}

Springer Nature remains neutral with regard to jurisdictional claims in published maps and institutional affiliations.
Ready to submit your research? Choose BMC and benefit from:

- fast, convenient online submission

- thorough peer review by experienced researchers in your field

- rapid publication on acceptance

- support for research data, including large and complex data types

- gold Open Access which fosters wider collaboration and increased citations

- maximum visibility for your research: over $100 \mathrm{M}$ website views per year

At BMC, research is always in progress.

Learn more biomedcentral.com/submissions 